Research Article

\title{
The Optimal Reinsurance Strategy under Conditional Tail Expectation (CTE) and Wang's Premium Principle
}

\author{
Shaoyong Hu $(\mathbb{D}$, Xingguo $\mathrm{Hu}(\mathbb{D}$, and Jun $\mathrm{Hu}(\mathbb{D}$ \\ School of Finance, Jiangxi University of Finance and Economics, Nanchang 330013, Jiangxi, China \\ Correspondence should be addressed to Jun Hu; hoojun98@jxufe.edu.cn
}

Received 13 April 2021; Accepted 15 May 2021; Published 26 May 2021

Academic Editor: Jiaqin Wei

Copyright (C) 2021 Shaoyong Hu et al. This is an open access article distributed under the Creative Commons Attribution License, which permits unrestricted use, distribution, and reproduction in any medium, provided the original work is properly cited.

In this study, we take the conditional tail expectation (CTE) as the constraint condition and consider the optimal reinsurance issues under Wang's premium principle in general insurance contracts. With the confidence level and the distortion function in Wang's premium principle given by the insurer in advance, a threshold can be obtained. When the insurer's risk tolerance level is greater than this value, the optimal reinsurance is a proportional reinsurance in which the deductible equals to this value, else the optimal form of reinsurance is a stop-loss reinsurance. Corresponding numerical examples and economic explanations are also given.

\section{Introduction}

Reinsurance is an important tool for the insurer to manage risks; through reinsurance, the insurer can mitigate the underwriting risk, thereby facilitating more effective risk management. For more than half a century, academics have established lots of optimal reinsurance models from the perspective of insurers and have investigated optimal reinsurance strategies under different conditions. Borch [1] solved that, under the principle of expected premium and fixed reinsurance premiums, a stop-loss reinsurance is the optimal for an insurer if the objective function is to minimize the variance of the insurer's loss. Kenneth [2] also supposed that, under the principle of expected premiums, the optimal reinsurance that a risk-averse insurer desires to maximize the expected utility of his own wealth is a stop-loss reinsurance contract. With the rapid development of financial liberalization, a new risk measure approach VaR (value at risk) is widely employed by banks, insurance companies, other financial institutions, and market regulators. Cai and Tan [3] hypothesized that the form of insurance is a stop-loss reinsurance, and the minimality of gross loss of the insurer measured by VaR and CTE as the objective function, and the optimal deductible is calculated under the principle of expected premium. Hu et al. [4] researched the calculation of the optimal retention of stoploss reinsurance under the condition of incomplete information on the aggregate loss function of the insurance company, while minimizing the VaR risk metric, and contrasted with the results of Cai and Tan [3]. Kong et al. [5] studied the optimal reinsurance issues in which both insurers and reinsurers face risks and uncertainties under general premium principles. Chi [6] considered a type of premium principle $P_{(X)}=E[X]+g(\operatorname{var}(X))$ (where $g$ is an increasing function of $g(0)=0)$. This type of premium principle includes the variance premium principle and the standard deviation premium principle. Under this type of premium principle, Chi [6] measured the aggregate loss of the insurer by VaR and CTE and indicated that the stop-loss reinsurance with the upper bounded is optimal. While taking the principle of variance premium and standard deviation premium as an example, the calculation shows how to obtain the optimal deductible and the upper boundary of indemnity. Chi and Tan [7] considered another type of premium principle, including Wang's premium principle and Dutch premium principle, and utilized VaR and CTE to calculate the aggregate loss of the insurer, and the optimal reinsurance is still a stop-loss reinsurance with the upper boundary. Cai et al. [8] studied Pareto optimal problem of reinsurance counterparty under one category of 
risk measure and gave a proof for the TVaR measure method. Putri et al. [9] considered the fixed premium under the expected premium standard. In order to minimize the risk of the insurer under $\mathrm{VaR}$, they combined proportional reinsurance with stop loss reinsurance to solve the optimal reinsurance strategy. Liang and Young [10] researched the minimum probability of bankruptcy of insurance companies, and Matteo and Claudia [11] introduced time variables and considered the necessities to pay fixed costs when signing reinsurance contracts. Jiang et al. [12] considered the maximization of the interests of both insurer and reinsurer from the perspespectives of expected utility maximization and risk minimization, and they found that the layer reinsurance is optimal.

Before introducing the framework of this paper, we first introduce the following notations. Let the insurer's possible loss $X$ in the future periods of time be a nonnegative random variable, the probability density function is $f(x)$, and the survival function is $S_{X}(x)=P\{X>x\}$. In order to control the risk effectively, the insurer transfers the loss $I(X)$ to the reinsurer. In exchange for underwriting risks, the reinsurer charges the insurer a reinsurance premium $P_{X}$. There are many calculation criteria for reinsurance premiums, and one of the more commonly used premium principle is the expected premium principle. In this paper, the more general Wang's premium principle is applied. It was first proposed by Wang [13]; it comprises the net premium principle, quantile premium principle, and dual risk premium principle. It is defined as $P_{X}=\int_{0}^{+\infty} g\left(S_{X}(x)\right) \mathrm{d} x$, for $g:[0,1] \longrightarrow[0,1]$ is a nondecreasing concave function which satisfies $g(x) \geq x, g(0)=0$, and $g(1)=1$. After the loss $I(X)$ ceded by the insurer, its retained loss function is $R(X)=X-I(X)$. Now, the insurer wants to control its retained losses within a certain spectrum because the measurement method only considers the risk of a certain quantile while ignoring the tail risk, but CTE considers the expectation of the entire tail risk. They are defined as follows: for a random variable, given a confidence level $1-\alpha(0<\alpha<1), \mathrm{VaR}$ is defined as
$\operatorname{VaR}_{\alpha}(X)=\inf \{x: \operatorname{Pr}\{X>x\} \leq \alpha\}$ and CTE is defined as $\operatorname{CTE}_{\alpha}(X)=E\left[X \mid X \geq \operatorname{VaR}_{\alpha}(X)\right]$.

The model constructed in this paper aims to minimize the reinsurance premium purchased by the insurer while controlling the risk of the insurer within a certain spectrum. Among them, the insurer's risk measure is CTE under this risk measure, the maximum risk that the insurer can accept is $N$, that is, the constraint is $\operatorname{CTE}_{\alpha}(R(X)) \leq N$. The objective function is $\min P_{X}=\min \int_{0}^{f \infty} g\left(S_{I(X)}(x)\right) \mathrm{d} x$. Because the larger the insurer's retention risk, the smaller the corresponding ceded risk, which results in a smaller premium, so there is an optimal solution as $\operatorname{CTE}_{\alpha}(R(X))=N$.

\section{Optimal Reinsurance}

In general insurance contracts, there are stop-loss reinsurance, quota-share reinsurance, layer reinsurance, and proportional reinsurance. The reinsurance used in this paper can include all forms of general insurance contracts. The specific notation is as follows:

$$
\begin{aligned}
I(x) & =l_{1}(x-a)_{+}-l_{2} \\
(x-b)_{+} & = \begin{cases}0, & 0 \leq x<a, \\
l_{1}(x-a), & a \leq x<b, \\
l_{1}(x-a)-l_{2}(x-b), & x \geq b,\end{cases}
\end{aligned}
$$

where $\left(l_{1} \geq l_{2}\right)$.

Insurer's retention risk is

$$
\begin{aligned}
R(x) & =x-l_{1}(x-a)_{+}+l_{2} \\
(x-b)_{+} & = \begin{cases}x, & 0 \leq x<a, \\
x-l_{1}(x-a), & a \leq x<b, \\
x-l_{1}(x-a)+l_{2}(x-b), & x \geq b,\end{cases}
\end{aligned}
$$

where $\left(l_{1} \geq l_{2}\right)$.

When the insurer's ceded loss function has the form of (1), the corresponding premium expression can be simplified as follows:

$$
\begin{aligned}
P_{X} & =\int_{0}^{+\infty} g\left(S_{I(X)}(x)\right) \mathrm{d} x=\int_{0}^{+\infty} g(P\{I(X)>x\}) \mathrm{d} x \\
& =\int_{0}^{+\infty} g\left(P\{0>x, X<a\}+P\left\{l_{1}(X-a)>x, a \leq X<b\right\}+P\left\{l_{1}(X-a)-l_{2}(X-b)>x, X \geq b\right\}\right) \mathrm{d} x \\
& =\int_{0}^{l_{1}(b-a)} g\left(P\left\{X>\frac{x}{l_{1}}+a\right\}\right) \mathrm{d} x+\int_{l_{1}(x-a)}^{+\infty} g\left(P\left\{X>\frac{x+l_{1} a-l_{2} b}{l_{1}-l_{2}}\right\}\right) \mathrm{d} x \\
& =l_{1} \int_{a}^{+\infty} g\left(S_{X}(x)\right) \mathrm{d} x-l_{2} \int_{b}^{+\infty} g\left(S_{X}(x)\right) \mathrm{d} x .
\end{aligned}
$$


Combine the objective function and the constraint conditions together with the Lagrange function:

$$
\begin{aligned}
L\left(a, b, l_{1}, l_{2}, \lambda\right)= & \int_{0}^{+\infty} g\left(S_{I(X)}(x)\right) \mathrm{d} x+\lambda\left[\operatorname{CTE}_{\alpha}(R(X))-N\right] \\
= & l_{1} \int_{a}^{+\infty} g\left(S_{X}(x)\right) \mathrm{d} x-l_{2} \int_{b}^{+\infty} g\left(S_{X}(x)\right) \mathrm{d} x \\
& +\lambda\left[\operatorname{CTE}_{\alpha}(R(X))-N\right] .
\end{aligned}
$$

While solving this equation, because of the magnitude relationship between $a, b$ and $\operatorname{VaR}_{\alpha}(X)$ is unknown, the value of $\mathrm{CTE}_{\alpha}(R(X))$ is uncertain. Therefore, the discussion should be divided into the following three circumstances.

Case 1. $\operatorname{VaR}_{\alpha}(X) \geq b$ :

$$
\begin{aligned}
L\left(a, b, l_{1}, l_{2}, \lambda\right)= & l_{1} \int_{a}^{+\infty} g\left(S_{X}(x)\right) \mathrm{d} x-l_{2} \int_{b}^{+\infty} g\left(S_{X}(x)\right) \mathrm{d} x \\
& +\lambda\left[\frac{1}{\alpha} \int_{\operatorname{VaR}_{\alpha}(X)}^{+\infty}\left(x-l_{1}(x-a)+l_{2}(x-b)\right) f(x) \mathrm{d} x-N\right] .
\end{aligned}
$$

Solving the above equation, $\left\{\begin{array}{l}b=a \\ a=a_{0} \\ l_{1}-l_{2}=l \\ \lambda=g\left(S_{X}\left(a_{0}\right)\right)\end{array}\right.$.

The ceded loss function is $I(x)=l\left(x-a_{0}\right)_{+}$, where $a_{0}$ and $l$ satisfies:

$$
\begin{array}{r}
\int_{a_{0}}^{+\infty} g\left(S_{X}(x)\right) \mathrm{d} x-\frac{g\left(S_{X}\left(a_{0}\right)\right)}{\alpha} \int_{\operatorname{VaR}_{\alpha}(X)}^{+\infty}\left(x-a_{0}\right) f(x) \mathrm{d} x=0, \\
\int_{\operatorname{VaR}_{\alpha}(X)}^{+\infty}\left(x-l\left(x-a_{0}\right)\right) f(x) \mathrm{d} x=\alpha N .
\end{array}
$$

Because $l \in[0,1]$, the value range of $N$, $N \in\left[a_{0}, \operatorname{CTE}_{\alpha}(X)\right]$ can be calculated in equation (6). When $N<a_{0}$, since there is no stable point for solving the equation, only the boundary $l=1, a=N$ can be taken. At this time, the ceded loss function is that $I(x)=(x-N)_{+}$; when
$N \geq \operatorname{CTE}_{\alpha}(X) \geq \operatorname{CTE}_{\alpha}(R(X))$, the insurer can bear the risk without purchasing reinsurance, that is, the optimal strategy is no reinsurance, and the reinsurance premium is 0 .

Case 2. $a \leq \operatorname{VaR}_{\alpha}(X)<b$ :

$$
\begin{aligned}
& \begin{aligned}
& L\left(a, b, l_{1}, l_{2}, \lambda\right)= l_{1} \int_{a}^{+\infty} g\left(S_{X}(x)\right) \mathrm{d} x-l_{2} \int_{b}^{+\infty} g\left(S_{X}(x)\right) \mathrm{d} x \\
&+\lambda\left[\frac{1}{\alpha} \int_{\operatorname{VaR}_{\alpha}(X)}^{b}\left(x-l_{1}(x-a)\right) f(x) \mathrm{d} x+\frac{1}{\alpha} \int_{b}^{+\infty}\left(x-l_{1}(x-a)+l_{2}(x-b)\right) f(x) \mathrm{d} x-N\right] . \\
& \text { Solving the above equation, }\left\{\begin{array}{l}
b \longrightarrow+\infty \\
a=a_{0} \\
l_{1}=l \\
l_{2}=0 \\
\lambda=g\left(S_{X}\left(a_{0}\right)\right)
\end{array} \quad \text { The ceded } \quad \int_{a_{0}}^{+\infty} g\left(S_{X}(x)\right) \mathrm{d} x-\frac{g\left(S_{X}\left(a_{0}\right)\right)}{\alpha} \int_{\operatorname{VaR}_{\alpha}(X)}^{+\infty}\left(x-a_{0}\right) f(x) \mathrm{d} x=0,\right. \\
& \int_{\operatorname{VaR}_{\alpha}(X)}^{+\infty}\left(x-l\left(x-a_{0}\right)\right) f(x) \mathrm{d} x=\alpha N .
\end{aligned} \\
& \text { Sunction is } I(x)=l\left(x-a_{0}\right)_{+}, \text {where } a_{0} \text { and } l \text { satisfies }
\end{aligned}
$$


Because $l \in[0,1]$, the value range of $N$, $N \in\left[a_{0}, \mathrm{CTE}_{\alpha}(X)\right]$ can be calculated in equation (8). When $N<a_{0}$, since there is no stable point for solving the equation, only the boundary $l=1$ and $a=N$ can be taken. At this time, the ceded loss function is that $I(x)=(x-N)_{+}$when $N \geq \mathrm{CTE}_{\alpha}(X) \geq \mathrm{CTE}_{\alpha}(R(X))$, the insurer can bear the risk without purchasing reinsurance, that is, the optimal strategy is no reinsurance, and the reinsurance premium is 0 . The result is the same as in the first case.

Case 3. $a>\operatorname{VaR}_{\alpha}(X)$ :

$$
\begin{aligned}
L\left(a, b, l_{1}, l_{2}, \lambda\right)= & l_{1} \int_{a}^{+\infty} g\left(S_{X}(x)\right) \mathrm{d} x-l_{2} \int_{b}^{+\infty} g\left(S_{X}(x)\right) \mathrm{d} x \\
& +\lambda\left[\frac{1}{\alpha} \int_{\operatorname{VaR}_{\alpha}(X)}^{a} x f(x) \mathrm{d} x+\frac{1}{\alpha} \int_{a}^{b}\left(x-l_{1}(x-a)\right) f(x) \mathrm{d} x+\frac{1}{\alpha} \int_{b}^{+\infty}\left(x-l_{1}(x-a)+l_{2}(x-b)\right) f(x) \mathrm{d} x-N\right]
\end{aligned}
$$

Solving the above equation, we can get two sets of solutions:

(i) $a=b=T\left(N \geq \mathrm{CTE}_{\alpha}(X)\right)$, the insurer is no need to buy reinsurance

(ii) $\left\{\begin{array}{l}b=a \text { or } b \longrightarrow+\infty \\ a=a_{0} \\ l_{1}-l_{2}=l \\ \lambda=\alpha \cdot\left(g\left(S_{X}\left(a_{0}\right)\right) / S_{X}\left(a_{0}\right)\right)\end{array}\right.$, where $a_{0}$ and 1 satisfies $\int_{\operatorname{VaR}_{\alpha}(X)}^{+\infty}\left(x-l\left(x-a_{0}\right)\right) f(x) \mathrm{d} x=\alpha N\left(\mathrm{VaR}_{\alpha}\right.$ $\left.(X) \leq N<\operatorname{CTE}_{\alpha}(X)\right)$.

In this case, the optimal ceded loss function is $I(x)=l\left(x-a_{0}\right)_{+}$.

Remark 1. Especially, if the internal constant $g^{\prime \prime}(x)=0$ in $(0, \alpha)$ is established and if $\mathrm{N}$ and $g(x)$ are given, $P_{X}$ will be a constant value. $a, b, l_{1}, l_{2}$ only need to satisfy $\mathrm{CTE}_{\alpha}(R(X))=N$. At this time, $I(x)=l \quad\left(x-a_{0}\right)_{+}$ and $I(x)=(x-a)_{+}$that meet the condition $\mathrm{CTE}_{\alpha}(R(X))=$ $N$ both can be used as the optimal reinsurance strategy, and the value of reinsurance premiums is the same.

Theorem 1. For any given $N$ and $g(x)$, a special point $a_{0} \in\left[0, \operatorname{VaR}_{\alpha}(X)\right]$ can be calculated, whereas $a_{0}$ satisfies

$$
\int_{a_{0}}^{+\infty} g\left(S_{X}(x)\right) \mathrm{d} x-\frac{g\left(S_{X}\left(a_{0}\right)\right)}{\alpha} \int_{\operatorname{VaR}_{\alpha}(X)}^{+\infty}\left(x-a_{0}\right) f(x) \mathrm{d} x=0 .
$$

(i) $0 \leq N<a_{0}$ : the optimal ceded loss function is $I(x)=(x-N)_{+}$, that is, the optimal reinsurance is the stop-loss reinsurance with a deductible of $N$.

(ii) $a_{0} \leq N<\operatorname{CTE}_{\alpha}(X)$ : the optimal ceded loss function is $I(x)=l\left(x-a_{0}\right)_{+}$, where $a_{0}$ and $l$ satisfy the following constraints:

$$
\begin{aligned}
& \int_{a_{0}}^{+\infty} g\left(S_{X}(x)\right) \mathrm{d} x-\frac{g\left(S_{X}\left(a_{0}\right)\right)}{\alpha} \int_{\operatorname{VaR}_{\alpha}(X)}^{+\infty}\left(x-a_{0}\right) f(x) \mathrm{d} x=0, \\
& \int_{\operatorname{VaR}_{\alpha}(X)}^{+\infty}\left(x-l\left(x-a_{0}\right)\right) f(x) \mathrm{d} x=\alpha N .
\end{aligned}
$$

If the internal constant $g^{\prime \prime}(x)=0$ in $x \in(0, \alpha)$ is established, it can be satisfied. $a, b, l_{1}, l_{2}$ only need to satisfy $C T E_{\alpha}(R(X))=N$. At this time, the ceded loss function $I(x)=l\left(x-a_{0}\right)_{+}, I(x)=(x-a)_{+}$ that meet the condition $\operatorname{CTE}_{\alpha}(R(X))=N$ can be used as the optimal reinsurance strategy.

(iii) $N \geq \mathrm{CTE}_{\alpha}(X)$ : in this situation, the insurer can bear the risk without purchasing reinsurance contract, so the optimal strategy is not to buy reinsurance, and the reinsurance premium is zero.

Remark 2. Especially, when $g(x)=x$, at that time, Wang's premium principle altered to the net premium principle. Calculating the following formula $\int_{a_{0}}^{+\infty} g\left(S_{X}(x)\right)$ $\mathrm{d} x-\left(g\left(S_{X}\left(a_{0}\right)\right) / \alpha\right) \int_{\operatorname{VaR}_{\alpha}(X)}^{+\infty}\left(x-a_{0}\right) f(x) \mathrm{d} x=0$ can be solved as $a_{0}=\operatorname{VaR}_{\alpha}(X)$, for a $N \in\left[0, \operatorname{CTE}_{\alpha}(X)\right]$ which is given by the insurer, and $I(x)=(x-a)_{+}$can be selected as the optimal reinsurance strategy. This result is consistent with the conclusion in [14].

\section{Numerical Examples}

Assume that the insurer's loss variable $X$ obeys the Pareto distribution. For any $x \geq 0$, the corresponding survival function

$$
S_{X}(x)=\left(\frac{200}{200+x}\right)^{3}, \quad x \geq 0 .
$$

The mean value of the variable $X$ is 100 . We assume that the confidence level of the insurance company to measure the risk 
TABle 1: When $g(x)=1-(1-x)^{3}$, the ceded functions and premiums correspond to different values of $N$.

\begin{tabular}{lcccccc}
\hline$\alpha$ & $\mathrm{VaR}_{\alpha}(X)$ & $\mathrm{CTE}_{\alpha}(X)$ & $N$ & $a$ & $l$ & $P$ \\
\hline \multirow{3}{*}{0.01} & \multirow{2}{*}{728.32} & \multirow{2}{*}{1144.48} & 726.44 & 726.44 & 1 & 13.93 \\
& & & 900 & 726.44 & 0.63 & 8.74 \\
\hline \multirow{3}{*}{0.025} & \multirow{2}{*}{483.99} & \multirow{2}{*}{825.99} & 480.48 & 480.48 & 1 & 25.65 \\
& & & 700 & 480.48 & 0.65 & 16.78 \\
\hline \multirow{3}{*}{0.1} & \multirow{2}{*}{230.89} & \multirow{2}{*}{441.53} & 221.82 & 221.82 & 1 & 64.78 \\
& & & 300 & 221.82 & 0.65 & 42.11 \\
\hline
\end{tabular}

TABle 2: When $g(x)=x$, the ceded functions and premiums correspond to different values of $N$.

\begin{tabular}{lcccccc}
\hline$\alpha$ & $\mathrm{VaR}_{\alpha}(X)$ & $\mathrm{CTE}_{\alpha}(X)$ & $N$ & $a$ & $l$ & $P$ \\
\hline \multirow{3}{*}{0.01} & \multirow{2}{*}{728.32} & \multirow{2}{*}{1144.48} & 726.44 & 726.44 & 1 & 4.66 \\
& & & 900 & $970(726)$ & $1(0.63)$ & 2.92 \\
\hline \multirow{3}{*}{0.025} & \multirow{2}{*}{483.99} & \multirow{2}{*}{825.99} & 480.48 & 480.48 & 1 & 8.64 \\
& & & 700 & $927(480)$ & $1(0.37)$ & 3.15 \\
\hline \multirow{3}{*}{0.1} & \multirow{2}{*}{230.89} & \multirow{2}{*}{441.53} & 221.84 & 221.82 & 1 & 22.48 \\
& & & 300 & $323(222)$ & $1(0.65)$ & 14.63 \\
\hline
\end{tabular}

is $1-\alpha$; according to the insurer's different confidence levels, when the retained risk is less than the insurer's maximum risk tolerance, solve the reinsurance strategy when the reinsurance premium is the smallest. Since the different distortion functions of Wang's premium can get the optimal ceded loss function under the corresponding premium principles, this paper considers the following two distortion functions:

(1) When $g(x)=1-(1-x)^{3}$, then $P_{X}$ is the dual risk premium principle

(2) When $g(x)=x$, then $P_{X}$ is the principle of net premium

According to the above two premium principles, Theorem 1 and $(\alpha, N)$ under different risk levels, the optimal reinsurance form can be obtained, as shown in Tables 1 and 2 .

When $g(x)=x$, the proportional coefficient $l$ can always get 1 , so the expression of the optimal ceded loss function is $I(x)=(x-a)_{+}$, when $0 \leq N \leq \operatorname{VaR}_{\alpha}(X), a=N$, whereas $\operatorname{VaR}_{\alpha}(X) \leq N \leq \operatorname{CTE}_{\alpha}(X)$, and there was $\int_{\operatorname{VaR}_{\alpha}(X)}^{a}$ $x f(x) \mathrm{d} x+\int_{a}^{+\infty}$ a $f(x) \mathrm{d} x=\alpha N$.

Since the expected premium principle is similar with the net premium principle, the net premium is merely multiplied by a coefficient. By contrasting different risk levels, it can be discovered that the premium of the dual risk premium principle is significantly higher in various situations.

\section{Conclusion}

The purpose of this paper is to control the insurer's risk level within his own capacity and choose a reinsurance contract that minimizes the reinsurance premium. In this paper, a threshold can be obtained based on the distortion function in Wang's premium principle and the insurer's confidence level. When the insurer's risk tolerance level $N$ is less than this value, the insurer will choose a stop-loss reinsurance with a deductible of $N$, and when the insurer's risk tolerance level $N$ is greater than this threshold, the insurer will choose a proportional stop-loss reinsurance with this threshold as the deductible. With deliberate consideration, this conclusion is reasonable. Since Wang's premium calculation guidelines charge higher premiums for tail risk, when the insurer's risk tolerance is large, the tail risk will not be fully ceded, but a proportional stop-loss reinsurance will be considered. When the insurer's risk tolerance is low, even if the insurer knows that the reinsurance premium for tail risk is high, the insurer still must choose reinsurance to control the risk.

\section{Data Availability}

No data were used to support this study.

\section{Conflicts of Interest}

The authors declare that they have no conflicts of interest.

\section{Acknowledgments}

The authors are grateful to the support from the National Natural Science Foundation of China (NSFC no. 71561012), the MOE (Ministry of Education in China) Project of Humanities and Social Sciences (20YJA790062), the Jiangxi Province Social Science Planning Project (19YJ12), the Jiangxi Province Education Department Science and Technology Project (GJJ180245 and GJJ170327), and The Humanities and Social Sciences Key Research Base Project of Universities in Jiangxi Province (JD18094).

\section{References}

[1] K. Borch, "An attempt to determine the optimum amount of stop loss reinsurance," Transactions of the 16th International Congress of Actuaries, vol. 1, pp. 597-610, 1960.

[2] K. J. Arrow, "Uncertainty and the welfare economics of medical care," The American Economic Review, Duke University Press, vol. 53, no. 5, , pp. 941-973, Durham, USA, 1963.

[3] J. Cai and K. S. Tan, "Optimal retention for a stop-loss reinsurance under the VaR and CTE risk measures," ASTIN Bulletin, vol. 37, no. 1, pp. 92-112, 2007.

[4] X. Hu, H. Yang, and L. Zhang, "Optimal retention for a stoploss reinsurance with incomplete information," Insurance Mathematics and Economics, vol. 65, no. 2, pp. 15-21, 2015.

[5] D. Kong, L. Liu, and Y. Wu, "Optimal reinsurance under risk and uncertainty on orlicz hearts," Insurance Mathematics and Economics, vol. 81, pp. 108-116, 2017.

[6] Y. Chi, "Optimal reinsurance under variance related premium principles," Insurance Mathematics and Economics, vol. 51, no. 2, pp. 310-322, 2015.

[7] Y. Chi and K. S. Tan, "Optimal reinsurance with general premium principles," Insurance Mathematics and Economics, vol. 52, no. 2, pp. 180-189, 2013. 
[8] J. Cai, H. Liu, and R. Wang, "Pareto-optimal reinsurance arrangements under general model settings," Insurance Mathematics and Economics, vol. 77, pp. 24-37, 2017.

[9] A. D. Putri, S. Nurrohmah, and I. Fithriani, "Quota-share and stop-loss reinsurance combination based on value-at-risk (VaR) optimization," Journal of Physics: Conference Series, vol. 1725 , no. 1,2021

[10] X. Liang and V. R. Young, "Minimizing the probability of ruin: optimal per-loss reinsurance," Insurance Mathematics and Economics, vol. 82, pp. 181-190, 2018.

[11] B. Matteo and C. Claudia, "Optimal reinsurance problem under fixed cost and exponential preferences," Mathematics, vol. 9 , no. 4 , p. 295,2021

[12] W. Jiang, H. Hong, and J. Ren, "Pareto-optimal reinsurance policies with maximal synergy," Insurance Mathematics and Economics, vol. 96, pp. 185-198, 2021.

[13] S. Wang, "Premium calculation by transforming the layer premium density," ASTIN Bulletin, vol. 26, no. 1, pp. 71-92, 1996.

[14] Z. Gao and M. Zhang, "The choice of optimal insurance contract under the constraint of tail condition expectation," Statistics and Decision, vol. 3, pp. 37-40, 2010. 